\title{
Organic solutes in Rubrobacter xylanophilus: the first example of di-myo-inositol-phosphate in a thermophile
}

\author{
Nuno Empadinhas $\cdot$ Vítor Mendes $\cdot$ Catarina Simões · \\ Maria S. Santos · Ana Mingote · Pedro Lamosa • \\ Helena Santos $\cdot$ Milton S. da Costa
}

Received: 23 February 2007/ Accepted: 3 April 2007/Published online: 18 May 2007

(C) Springer 2007

\begin{abstract}
The thermophilic and halotolerant nature of Rubrobacter xylanophilus led us to investigate the accumulation of compatible solutes in this member of the deepest lineage of the Phylum Actinobacteria. Trehalose and mannosylglycerate (MG) were the major compounds accumulated under all conditions examined, including those for optimal growth. The addition of $\mathrm{NaCl}$ to a complex medium and a defined medium had a slight or negligible effect on the accumulation of these compatible solutes. Glycine betaine, di-myo-inositol-phosphate (DIP), a new phosphodiester compound, identified as di- $N$-acetylglucosamine phosphate and glutamate were also detected but in low or trace levels. DIP was always present, except at the highest salinity examined $(5 \% \mathrm{NaCl})$ and at the lowest temperature tested $\left(43^{\circ} \mathrm{C}\right)$. Nevertheless, the levels of DIP increased with the growth temperature. This is the first report of MG and DIP in an actinobacterium and includes the identification of the new solute di- $N$-acetylglucosamine phosphate.
\end{abstract}

Communicated by G. Antranikian.

N. Empadinhas · V. Mendes · C. Simões ·

M. S. Santos

Centro de Neurociências e Biologia Celular,

Departamento de Zoologia, Universidade de Coimbra, 3004-517 Coimbra, Portugal

A. Mingote $\cdot$ P. Lamosa $\cdot$ H. Santos

Instituto de Tecnologia Química e Biológica,

Universidade Nova de Lisboa, Rua da Quinta Grande 6,

Apartado 127, 2780-156 Oeiras, Portugal

M. S. da Costa ( $\square)$

Departamento de Bioquímica, Universidade de Coimbra, 3001-401 Coimbra, Portugal

e-mail: milton@ci.uc.pt
Keywords Rubrobacter xylanophilus - Organic solutes · Mannosylglycerate $\cdot$ Di-myo-inositol-phosphate

\section{Introduction}

Some microorganisms have developed specific adaptations to extraordinarily inhospitable environments; however, most organisms appear to react to stresses, within inherent limits, by mobilizing available resources crucial for their survival. Hyperosmotic shock, for example, induces the accumulation of small organic molecules, designated compatible solutes (Brown 1976). More rarely, potassium chloride is accumulated and in some cases potassium and negatively charged compatible solutes are both involved in osmotic adjustment (da Costa et al. 1998). Increasing evidence supports an additional talent of compatible solutes in the stabilization of the native folding of enzymes (Lamosa et al. 2000). Some compatible solutes, namely trehalose are also involved in the response to other stress conditions induced by temperature, desiccation, freezing, oxygen deprivation, nutrient starvation, toxic compounds and oxidative agents, and are viewed as general stress protectants (Hoelzle and Streeter 1990; da Costa et al. 1998; Santos and da Costa 2002; Elbein et al. 2003).

Trehalose, glycine betaine and glutamate are widespread in nature while other organic solutes are restricted to a few groups of organisms. Cyclic-2,3-bisphosphoglycerate (cBPG), for example, has been found only in methanogenic archaea (Roberts 2004); diglycerol phosphate (DGP) is restricted to Archaeoglobus spp. and di-myo-inositolphosphate (DIP) has only been identified in hyperthermophilic archaea and bacteria which suggests a role in thermoprotection (Santos and da Costa 2002). Mannosylglycerate (MG), on the other hand, was initially discovered 
in a group of red algae and later in several thermophilic bacteria and hyperthermophilic archaea where it behaves like a typical compatible solute, leading to the hypothesis that MG is an archetypal solute of prokaryotes living at high growth temperatures (Bouveng et al. 1955; Santos and da Costa 2002). However, the accumulation of MG in marine red algae and the discovery of genes for the synthesis of MG in mesophilic bacteria and in archaeal metagenomes obtained from low temperature environments argue against the hypothesis of a role of MG exclusively in the adaptation to thermal environments (Empadinhas and da Costa 2006).

The bacteria of the genus Rubrobacter represent the most ancient lineage of the phylum Actinobacteria (High $\mathrm{G}+\mathrm{C}$ Gram-positive bacteria) known and are assigned to three known species, namely $R$. radiotolerans, $R$. taiwanensis and $R$. xylanophilus with optimum growth temperatures of 45,55 , and $60^{\circ} \mathrm{C}$, respectively (Carreto et al. 1996; Chen et al. 2004; Suzuki et al. 1988). All strains examined of Rubrobacter are gamma-radiation resistant and $R$. xylanophilus is, not only the most thermophilic actinobacterium known, but also the only true extremely radiation-resistant thermophile (Suzuki et al. 1988; Ferreira et al. 1999; Chen et al. 2004). R. xylanophilus is also halotolerant growing in medium containing up to $6 \% \mathrm{NaCl}$. Because it is not common to find an organism with these characteristics, it was deemed important to investigate the effect of salinity and temperature on the diversity and on levels of compatible solutes.

\section{Materials and methods}

Strains and culture conditions

The type strain of $R$. xylanophilus PRD- ${ }^{\mathrm{T}}$ (=DSM $9941^{\mathrm{T}}$ ) was from our collection. The organism was grown aerobically in Thermus medium (Williams and da Costa 1992) containing tryptone $\left(1.0 \mathrm{~g} \mathrm{~L}^{-1}\right)$ and yeast extract $\left(1.0 \mathrm{~g} \mathrm{~L}^{-1}\right)$, and in a minimal medium ( $\mathrm{M}$ medium) containing basal salts of Degryse medium 162 (Degryse et al. 1978), yeast extract $\left(0.1 \mathrm{~g} \mathrm{~L}^{-1}\right)$, glucose $\left(5.0 \mathrm{~g} \mathrm{~L}^{-1}\right)$ and $\mathrm{NH}_{4} \mathrm{Cl}$ $\left(2.0 \mathrm{~g} \mathrm{~L}^{-1}\right)$; the $\mathrm{pH}$ was adjusted to 8.2. To examine the effect of osmotic stress on the accumulation of intracellular solutes, cells were grown at $60^{\circ} \mathrm{C}$ in medium with $2.5,4.0$ or $5.0 \% \mathrm{NaCl}(\mathrm{w} / \mathrm{v})$, or without $\mathrm{NaCl}$. Cultures were also grown at $43,50,60$ and $67^{\circ} \mathrm{C}$ in medium without $\mathrm{NaCl}$ to examine the effect of the growth temperature on the accumulation of intracellular solutes. Growth of the organisms was performed in 1-liter metal-capped Erlenmeyer flasks containing $200 \mathrm{ml}$ of medium in a reciprocalwater bath shaker (120 rpm) and monitored by measuring the turbidity of the cultures at $600 \mathrm{~nm}$. Cells were harvested during the mid-exponential phase of growth by centrifugation $\left(8,000 \times g, 4^{\circ} \mathrm{C}\right.$ for $\left.10 \mathrm{~min}\right)$ and washed twice with a $\mathrm{NaCl}$ solution identical in concentration to that of the medium in which the cells were grown.

Extraction of organic solutes

Cell pellets were extracted twice with boiling $80 \%$ ethanol and the extracts were freeze-dried as described previously (Silva et al. 1999). The dry weight of cells was determined by filtering cell suspensions under vacuum, through dried pre-weighted $0.22 \mu \mathrm{m}$ pore size filters (Gelman). The filters were dried for $24 \mathrm{~h}$ at $70^{\circ} \mathrm{C}$ and weighted again.

\section{Purification of the new phosphorylated sugar compound}

Ethanol extracts of $R$. xylanophilus cells containing the unidentified compound were loaded on to a QAE-Sephadex column (Pharmacia, Uppsala, Sweden) equilibrated at pH 9.8 with $5 \mathrm{mM}$ sodium carbonate buffer. The sample was eluted applying a linear gradient between $5 \mathrm{mM}$ and $1 \mathrm{M}$ of $\mathrm{NaHCO}_{3}$ buffer. Fractions were collected and analyzed by NMR. The fractions containing the new compound were pooled, the buffer removed in an activated Dowex $50 \mathrm{~W}-\mathrm{X} 8$ column and freeze dried. The purest sample was dissolved in a mixture of dichloromethane: methanol: $25 \%$ ammonia $(6: 10: 5, \mathrm{v} / \mathrm{v} / \mathrm{v})$, centrifuged and the supernatant applied to a silica gel column equilibrated with the same solvent. After removing the solvent by evaporation, the fractions were analyzed by NMR.

\section{NMR spectroscopy}

Freeze-dried extracts were analyzed by nuclear magnetic resonance (NMR) as described previously (Silva et al. 1999). The quantification of solutes was determined by comparing the intensities of resonances of the compounds in the sample with the one from a concentration standard, in our case formate. All spectra were acquired on a Bruker DRX500 spectrometer. For quantification purposes, ${ }^{1} \mathrm{H}$ NMR spectra were acquired with water presaturation, $6 \mu \mathrm{s}$ pulse width $\left(60^{\circ}\right.$ flip angle) and a repetition delay of $60 \mathrm{~s}$. Chemical shifts were relative to 3-(trimethylsilyl)propanesulfonic acid (sodium salt). Formate was added as an internal concentration standard. ${ }^{1} \mathrm{H}-\mathrm{NMR}$ spectra were also acquired with selective irradiation of the phosphorous signal at $-2.73 \mathrm{ppm}$.

${ }^{31} \mathrm{P}-\mathrm{NMR}$ spectra were recorded at $202.45 \mathrm{MHz}$ using a broadband inverse detection 5-mm probe head. Broadband proton decoupling was applied during the acquisition time only, using the wideband alternating-phase low-power technique for zero-residue splitting sequence (WALTZ); 
for selective irradiation, spectra were acquired with continuous irradiation of the desired proton signal.

Two-dimensional spectra were performed using standard Bruker pulse programs. Proton-homonuclear shift correlation spectroscopy (COSY) and total-correlation spectroscopy (TOCSY) were acquired collecting $4096\left(t_{2}\right) \times 512\left(t_{1}\right)$ data points; while in ${ }^{1} \mathrm{H}-{ }^{13} \mathrm{C}$ and ${ }^{1} \mathrm{H}-{ }^{31} \mathrm{P}$ heteronuclear multiple quantum coherence spectra (HMQC) $4096\left(t_{2}\right) \times 256\left(t_{1}\right)$ data points were collected (Bax and Summers 1986). Delays of $3.5 \mathrm{~ms}$ and $65 \mathrm{~ms}$ were used for evolution of ${ }^{1} J_{\mathrm{XH}}$ in the carbon and phosphorous correlation spectra, respectively.

\section{Mass spectrometry}

Mass spectra were acquired on a LCQ advantage ion trap mass spectrometer from ThermoFinnigan (San Jose, CA, USA) equipped with an electrospray ionization interface operated in the negative mode. Samples were injected at $300^{\circ} \mathrm{C}$ and at $-33 \mathrm{~V}$ in $50 \%$ methanol/ $0.1 \%$ formic acid.

\section{High-performance liquid chromatography (HPLC)}

Amino acids were analyzed in a Gilson-ASTED system according to the manufacturer's instructions. The amino acid derivatives resulting from the pre-column derivatization with orthophthaldeyde/2-mercaptoethanol were separated on a Spherisorb ODS column (particle size, $5 \mu \mathrm{m}$; $150 \mathrm{~mm}$ long, $4.6 \mathrm{~mm} \mathrm{ID),} \mathrm{at} \mathrm{a} \mathrm{flow} \mathrm{rate} \mathrm{of} 2.5 \mathrm{~mL} / \mathrm{min}$, using a ternary solvent system: buffer A (250 mM sodium phosphate, 15\%; $200 \mathrm{mM}$ propionic acid, 20\%; acetonitrile, 7\%; DMSO, 3\%; pH 6.2); buffer B (acetonitrile, 40\%; methanol, 33\%; DMSO, 7\%); and buffer C (250 mM sodium phosphate, 25\%; $250 \mathrm{mM}$ propionic acid, 20\%; acetonitrile, 7\%; DMSO, 3\%; pH 5.5). The effluent was monitored by a fluorescent detector (Gilson, model 121; excitation and emission wavelengths at 340 and $410 \mathrm{~nm}$, respectively). The Gilson system software performed the integration of the amino acid peak area and further calculations, and quantification was allowed by running standard amino acids solutions in the same conditions.

\section{Results}

Identification and quantification of the organic solutes

${ }^{1} \mathrm{H}-\mathrm{NMR}$ spectra of the cell extracts were recorded and the signals compared with those from the literature. This analysis allowed the detection of mannosylglycerate (MG), and trehalose, as major compounds, as well as lower amounts of glycine betaine, di-myo-inositol-phosphate (DIP) and very low levels of an unidentified compound with a signal in the region characteristic of the anomeric protons of hexoses. The analyses of the ${ }^{31} \mathrm{P}-\mathrm{NMR}$ spectrum revealed the presence of a resonance assigned to DIP and another signal at $-2.73 \mathrm{ppm}$ that could not be immediately assigned and was therefore suspected to belong to the same unidentified compound. Due to the small relative amount of the new solute, we resorted to chromatography to purify it. The first chromatographic step gave origin to two partially purified fractions, one containing the new solute and DIP, and another one with the new compound, as well as DIP and MG. Since the purification was followed by NMR it was possible to observe that the relative intensities of the anomeric signal in the ${ }^{1} \mathrm{H}$ and the unidentified ${ }^{31} \mathrm{P}$ resonance was constant in all the samples, strongly supporting the view that the new sugar compound was phosphorylated. This assumption was confirmed by the acquisition of a ${ }^{1} \mathrm{H}-^{31} \mathrm{P}$ correlation HMQC spectrum that revealed a strong signal between the phosphorous and the anomeric signal at $5.46 \mathrm{ppm}$, and a lesser signal at the same phosphorous resonance to a proton signal at $3.99 \mathrm{ppm}$, interpreted as the proton at position 2 of the sugar moiety. This interpretation was confirmed by a homonuclear proton correlation spectrum (COSY) (Fig. 1). The structure of the phosphorous signal is that of a triplet of triplets with coupling constants of $J_{\mathrm{PH}} 7.44$ and $3.10 \mathrm{~Hz}$, indicating that it is split by two groups of two protons. Since the HMQC showed connectivities only to positions 1 and 2 of the sugar moiety, there has to be two sugar moieties splitting the phosphorous resonance i.e. the molecule is a symmetric di-sugar phosphodiester. This deduction was confirmed by the acquisition of phosphorous spectra with selective irradiation of the sugar protons at positions 1 and 2 giving rise to distinct triplets, and by the fact that the proton/phosphorous relative intensities of the new compound were the same as DIP (a phosphodiester).

The fraction containing only the new solute and DIP was applied to a silica gel column that separated the two compounds. The ${ }^{1} \mathrm{H}$ spectrum of this sample revealed a singlet at $2.06 \mathrm{ppm}$ with a 3:1 intensity ratio to the anomeric signal, which led us to suspect the presence of an $\mathrm{N}$ acetyl substituent. To confirm this hypothesis, the solvent in the NMR sample was exchanged from $100 \% \mathrm{D}_{2} \mathrm{O}$ to $10 \% \mathrm{D}_{2} \mathrm{O}$ leading to the appearance of one extra proton resonance in the amide region of the spectrum. The COSY spectrum (Fig. 1) permitted to assign all proton resonances of the hexose and showed that the acetyl group was bound to position 2. The acquisition of a ${ }^{1} \mathrm{H}^{-13} \mathrm{C} \mathrm{HMQC}$ allowed the assignment of the carbon resonances (Table 1). Analysis of the coupling constants (Table 1) revealed that the hexose was glucose in the $\alpha$-pyranosyl configuration, therefore, establishing the structure of the new compound as di- $N$-acetyl-glucosamine phosphate (Fig. 1). This identification was further confirmed by mass spectrometry. The 
Fig. 1 Proton homonuclear correlation spectrum (COSY) of the new compound in $90 \% \mathrm{H}_{2} \mathrm{O}$. A schematic representation of the new phosphodiester compound, di- $N$-acetylglucosamine phosphate (DAGAP), is depicted as an inset
Table 1 NMR parameters of di- $N$-acetyl-glucosamine phosphate

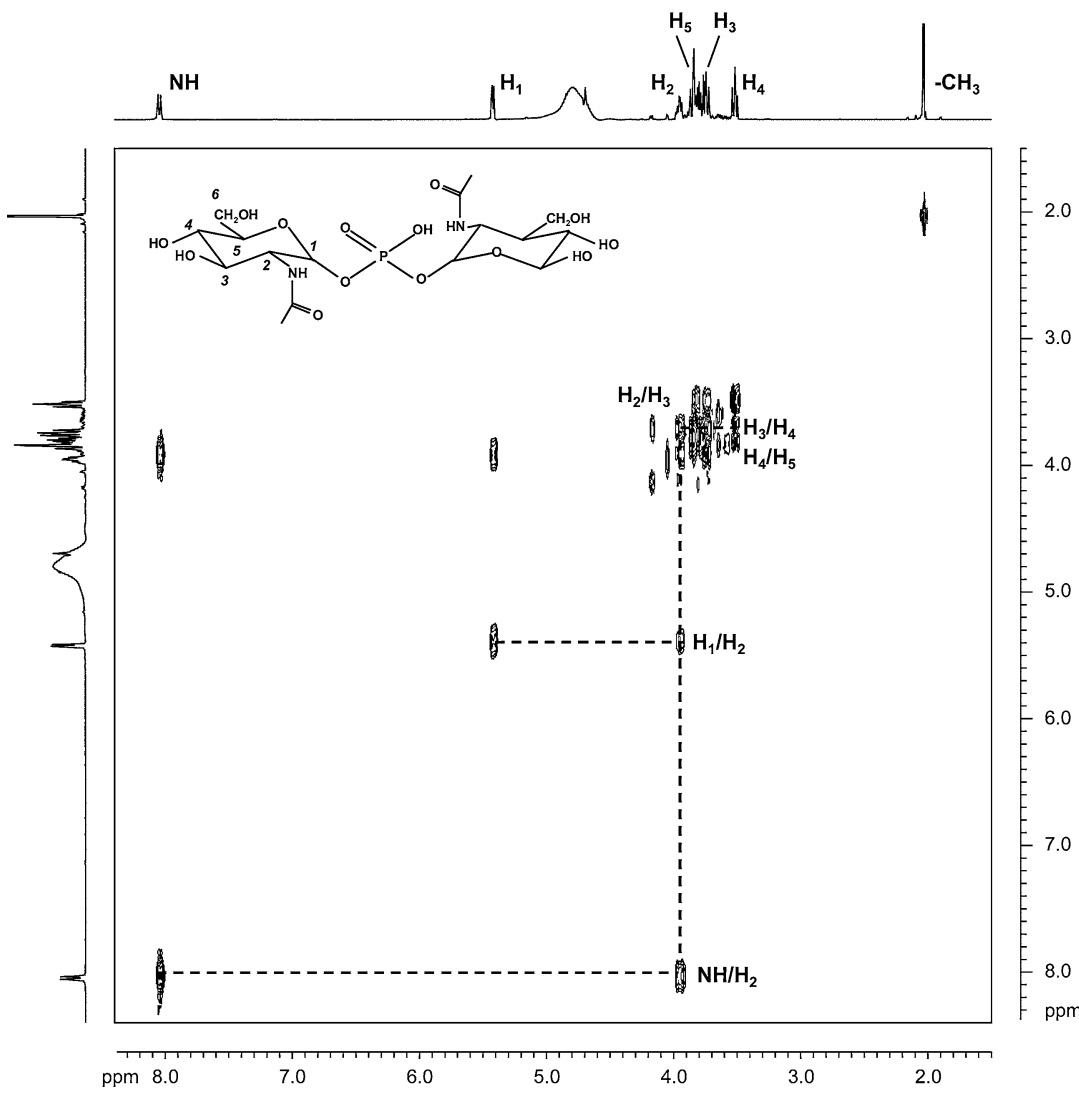

\begin{tabular}{|c|c|c|c|c|c|}
\hline \multirow[t]{2}{*}{ Moiety } & \multirow{2}{*}{$\begin{array}{l}{ }^{13} \mathrm{C}-\mathrm{NMR} \\
\delta(\mathrm{ppm})\end{array}$} & \multicolumn{2}{|l|}{${ }^{1} \mathrm{H}-\mathrm{NMR}$} & \multicolumn{2}{|l|}{${ }^{31} \mathrm{P}-\mathrm{NMR}$} \\
\hline & & $\delta(\mathrm{ppm})$ & ${ }^{3} J_{\mathrm{HH}}(\mathrm{Hz})$ & $\delta(\mathrm{ppm})$ & ${ }^{3} J_{\mathrm{PH}}(\mathrm{Hz})$ \\
\hline \multicolumn{6}{|l|}{ Glucosyl } \\
\hline $\mathrm{C}_{1}$ & 100.38 & 5.46 & ${ }^{3} J_{1,2}=3.42$ & & ${ }^{3} J_{\mathrm{H} 1}=7.44$ \\
\hline $\mathrm{C}_{2}$ & 59.87 & 3.99 & ${ }^{3} J_{2,3}=9.54$ & & ${ }^{3} J_{\mathrm{H} 2}=3.10$ \\
\hline $\mathrm{C}_{3}$ & 76.81 & 3.77 & ${ }^{3} J_{3,4}=10.03$ & & \\
\hline $\mathrm{C}_{4}$ & 75.83 & 3.55 & ${ }^{3} J_{4,5}=9.29$ & & \\
\hline $\mathrm{C}_{5}$ & 79.27 & 3.86 & ND & & \\
\hline $\mathrm{C}_{6}$ & 66.74 & $3.88 ; 3.81$ & ND & & \\
\hline Amine & & 8.09 & ${ }^{3} J_{\mathrm{NH}, 2}=9.05$ & & \\
\hline Methyl & 28.20 & 2.06 & & & \\
\hline Phosphorous & & & & -2.73 & \\
\hline
\end{tabular}

$N D$ not determined

mass spectrum of the pure compound revealed a signal with a $\mathrm{m} / \mathrm{z}$ of 503.9 corresponding to the expected mass of di- $N$-acetyl-glucosamine phosphate.

Effect of salinity and temperature on the growth rate and on the accumulation of intracellular organic solutes

The organism was halotolerant, with an optimal growth rate in medium without added $\mathrm{NaCl}$, but was able to maintain consistent growth in media containing up to $5 \%$
$\mathrm{NaCl}$. The organism had an optimum growth around $60^{\circ} \mathrm{C}$, growing at temperatures as low as $43^{\circ} \mathrm{C}$ and as high as $67^{\circ} \mathrm{C}$. In the $\mathrm{M}$ medium the growth rates were slightly lower than those obtained in Thermus medium and decreased more rapidly at supra-optimal salinity and temperature (Fig. 2). Moreover, growth could not be measured in $\mathrm{M}$ medium at $43^{\circ} \mathrm{C}$ (Fig. 2d).

The total intracellular solute pools in cells grown in $\mathrm{M}$ medium were higher than those measured in cells grown in Thermus medium, under all conditions tested, but 
Fig. 2 Growth rate of $R$. xylanophilus (filled square with line) in Thermus medium $(\mathbf{a}, \mathbf{b})$ and $\mathrm{M}$ medium $(\mathbf{c}, \mathbf{d})$ and the effect of $\mathrm{NaCl}$ concentration $(\mathbf{a}, \mathbf{c})$ and temperature $(\mathbf{b}, \mathbf{d})$ on the accumulation of compatible solutes in cells harvested during mid-exponential phase of growth. Concentration of trehalose (open rectangle), mannosylglycerate (light shaded rectangle), di-myoinositol-phosphate (filled rectangle), glycine betaine (rectangle with vertical lines), and di- $N$-acetyl-glucosamine phosphate (rectangle with dots) were determined by NMR; glutamate (rectangle with horizontal lines) concentration was determined by HPLC
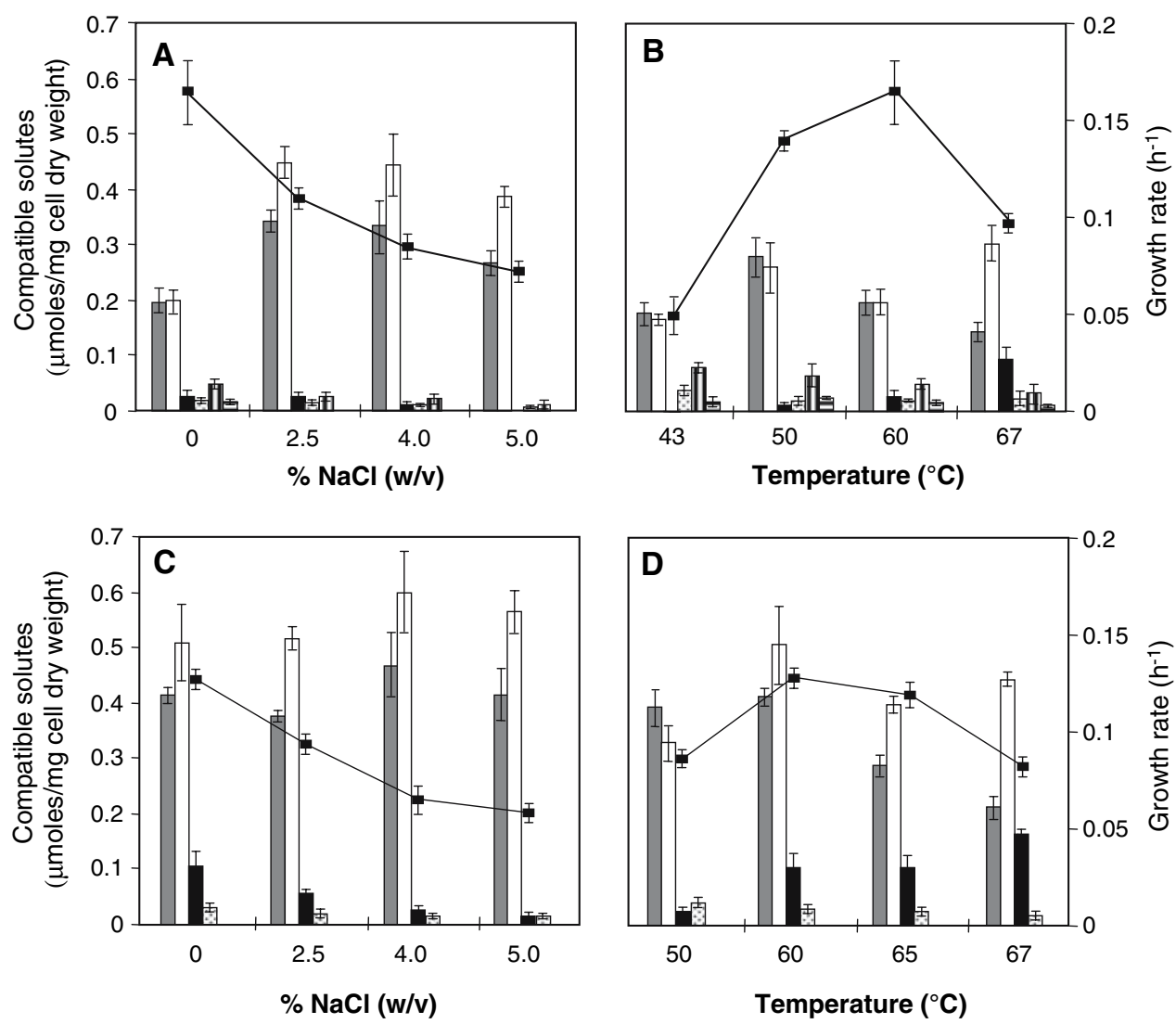

otherwise the levels of each solute varied little with salinity of the medium or growth temperature (Fig. 2). High solute pools were found under optimal growth conditions in $\mathrm{M}$ medium and Thermus medium without added $\mathrm{NaCl}$. The major organic solutes were, under all conditions examined, trehalose and MG. In Thermus medium, the increase in the salinity from 0 to $2.5 \% \mathrm{NaCl}$ resulted in an increase in the concentration of trehalose and MG, which remained fairly constant when the concentration of $\mathrm{NaCl}$ was raised to 4 and 5\% (Fig. 2a). In M medium, trehalose and MG concentrations did not change with salinity, but the concentration of DIP decreased significantly (Fig. 2c). DIP was not detected during growth at $43^{\circ} \mathrm{C}$ in Thermus medium, but otherwise increased with growth temperature, in both media, never reaching levels as high as trehalose and MG (Fig. $2 b$ and d). Amino acids, over $90 \%$ of which were glutamate, were always present in very low amounts that did not exceed $0.023 \mu \mathrm{mol}$ per $\mathrm{mg}$ of cell dry weight (Fig. 2a). The concentration of the newly identified solute, di- $\mathrm{N}$-acetylglucosamine phosphate, was always low and remained constant under all conditions tested. Glycine betaine was detected only in cells grown in Thermus medium and the highest concentration was at the lowest temperature tested (Fig 2b).

\section{Discussion}

The organic solutes of $R$. xylanophilus were always found in high levels in media without $\mathrm{NaCl}$ changing little during growth under salt and temperature stress, and thus making it difficult to clearly attribute a role to their accumulation. These solutes, must, by virtue of the intracellular levels, confer osmotic protection to the organism under salt stress. However, some Low G + C Gram-Positive Bacteria also accumulate high levels of compatible solutes, primarily amino acids, in medium containing low levels of $\mathrm{NaCl}$ (Poolman and Glasker 1998). Generally, glutamate, proline and ectoine are the most abundant compatible solutes of those Bacteria, while a few high $\mathrm{G}+\mathrm{C}$ Gram-Positive Bacteria (Phylum Actinobacteria) studied, namely corynebacteria and streptomycetes accumulate trehalose, proline, glutamine and alanine under osmotic stress (Shimakata and Minatogawa 2000; Wolf et al. 2003). These organisms as well as mycobacteria accumulate a low steady-state trehalose pool, and in some Streptomyces sp., trehalose is also involved in spore germination being implicated in their heat and desiccation resistance (McBride and Ensign 1987; Woodruff et al. 2004). However, the accumulation of high concentrations of sugar-based organic solutes in $R$. xylanophilus under 
non-stressing conditions and upon salt stress is, to our knowledge, unprecedented. The utilization of a minimal medium devoid of trehalose, demonstrates that $R$. xylanophilus, like Corynebacterium spp. and Mycobacterium spp. can synthesize this solute (De Smet et al. 2000; Wolf et al. 2003). The accumulation of MG by $R$. xylanophilus came somewhat as a surprise because this organism has a lower growth range than other thermophilic bacteria, namely T. thermophilus and Rhodothermus marinus, where MG serves as a compatible solute. In fact, we detected MG in cells from cultures of $R$. xylanophilus grown at $43^{\circ} \mathrm{C}$ which is the lowest growth temperature of any known bacterium that accumulates this compatible solute.

Glycine betaine (GB), a common compatible solute of mesophilic prokaryotes, was a minor solute in $R$. xylanophilus grown in Thermus medium, indicating that it was taken up, along with glutamate, from the yeast extract and tryptone. This solute cannot, however, have a role in osmotic adjustment in this organism because of the minor amounts accumulated. Moreover, betaine is not detected in (hyper)thermophilic bacteria or archaea, indicating that it is not used for osmotic adjustment in these organisms (Santos and da Costa 2002).

Interestingly, the pool of organic solutes in R. xylanophilus comprises two symmetrical phosphodiesters: the novel compound, di- $N$-acetyl-glucosamine phosphate (DAGAP), which was detected in minor amounts under all conditions examined, and di-myo-inositol phosphate, a polyol-phosphodiester widespread in hyperthermophilic archaea and bacteria, but never encountered in organisms with optimal growth temperatures below $80^{\circ} \mathrm{C}$ (Santos and da Costa 2002). The occurrence of DIP in $R$. $x y$ lanophilus, a thermophile with $T_{\text {opt }}=60^{\circ} \mathrm{C}$ was, therefore, unexpected. Moreover, the levels of DIP increased clearly with the growth temperature, a trend also observed in the hyperthermophiles examined (Santos and da Costa 2002).

It is noteworthy that all the phosphodiester solutes of hyperthermophiles reported to date, such as diglycerol phosphate, glycerol-phospho-myo-inositol, and di-mannosyl-di-myo-inositol phosphate are polyol derivatives, whereas the new solute identified here comprises a phosphate group linking two sugar moieties (Santos and da Costa 2002; Lamosa et al. 2006). The finding that the thermophilic bacterium $R$. xylanophilus accumulates DIP and MG leads us to speculate that the growth temperature range for the accumulation of these compatible solutes is more flexible than initially suspected.

Acknowledgments This work was supported by Fundação para a Ciência e a Tecnologia (FCT), Portugal, and FEDER, projects POCTI/BIO/42331/2001, POCI/BIA-MIC/56511/2004 and POCI/ BIA-PRO/57263/2004. N. Empadinhas and P. Lamosa acknowledge scholarships from FCT (SFRH/BPD/14828/2003 and SFRH/BPD/
26606/2006). We wish to thank Ana Coelho (Mass Spectrometry Service, ITQB, Oeiras) for performing the mass spectra.

\section{References}

Bax A, Summers MF (1986) ${ }^{1} \mathrm{H}$ and ${ }^{13} \mathrm{C}$ assignments from sensitivityenhanced detection of heteronuclear multiple-bond connectivity by 2D multiple quantum NMR. J Am Chem Soc 108:2093-2094

Bouveng H, Lindberg B, Wickberg B (1955) Low-molecular carbohydrates in algae. Structure of the glyceric acid mannoside from red algae. Acta Chem Scand 9:807-809

Brown AD (1976) Microbial water stress. Bacteriol Rev 40:803-846

Carreto L, Moore E, Nobre MF, Wait R, Riley PW, Sharp RJ, da Costa MS (1996) Rubrobacter xylanophilus sp. nov., a new thermophilic species isolated from a thermally polluted effluent. Int J Syst Bacteriol 46:460-465

Chen MY, Wu SH, Lin GH, Lu CP, Lin YT, Chang WC, Tsay SS (2004) Rubrobacter taiwanensis sp. nov., a novel thermophilic, radiation-resistant species isolated from hot springs. Int J Syst Evol Microbiol 54:1849-1855

da Costa MS, Santos H, Galinski EA (1998) An overview of the role and diversity of compatible solutes in Bacteria and Archaea. Adv Biochem Eng Biotechnol 61:117-153

De Smet KA, Weston A, Brown IN, Young DB, Robertson BD (2000) Three pathways for trehalose biosynthesis in mycobacteria. Microbiology 146:199-208

Degryse E, Glansdorff N, Pierard A (1978) A comparative analysis of extreme thermophilic bacteria belonging to the genus Thermus. Arch Microbiol 117:189-196

Elbein AD, Pan YT, Pastuszak I, Carroll D (2003) New insights on trehalose: a multifunctional molecule. Glycobiology 13:17R27R

Empadinhas N, da Costa MS (2006) Diversity and biosynthesis of compatible solutes in hyper/thermophiles. Int Microbiol 9:199_ 206

Ferreira AC, Nobre MF, Moore E, Rainey FA, Battista JR, da Costa MS (1999) Characterization and radiation resistance of new isolates of Rubrobacter radiotolerans and Rubrobacter xylanophilus. Extremophiles 3:235-238

Hoelzle I, Streeter JG (1990) Increased accumulation of trehalose in rhizobia cultured under 1\% Oxygen. Appl Environ Microbiol 56:3213-3215

Lamosa P, Burke A, Peist R, Huber R, Liu MY, Silva G, RodriguesPousada C, LeGall J, Maycock C, Santos H (2000) Thermostabilization of proteins by diglycerol phosphate, a new compatible solute from the hyperthermophile Archaeoglobus fulgidus. Appl Environ Microbiol 66:1974-1979

Lamosa P, Gonçalves LG, Rodrigues MV, Martins LO, Raven NDH, Santos H (2006) Occurrence of 1-Glyceryl-1-myo-inosityl-phosphate in hyperthermophiles. Appl Environ Microbiol 72:61696173

McBride MJ, JC Ensign (1987) Metabolism of endogenous trehalose by Streptomyces griseus spores and by spores or cells of other actinomycetes. J Bacteriol 169:5002-5007

Poolman B, Glaasker E (1998) Regulation of compatible solute accumulation in bacteria. Mol Microbiol 29:397-407

Roberts MF (2004) Osmoadaptation and osmoregulation in archaea: update 2004. Front Biosci 9:1999-2019

Santos H, da Costa MS (2002) Compatible solutes of organisms that live in hot saline environments. Environ Microbiol 4:501-509

Shimakata T, Minatogawa Y (2000) Essential role of trehalose in the synthesis and subsequent metabolism of corynomycolic acid in Corynebacterium matruchotii. Arch Biochem Biophys 380:331338 
Silva Z, Borges N, Martins LO, Wait R, da Costa MS, Santos H (1999) Combined effect of the growth temperature and salinity of the medium on the accumulation of compatible solutes by Rhodothermus marinus and Rhodothermus obamensis. Extremophiles 3:163-172

Suzuki K, Collins MD, Iijima E, Komagata K (1988) Chemotaxonomic characterization of a radiotolerant bacterium, Arthrobacter radiotolerans: description of Rubrobacter radiotolerans gen. nov., comb. nov. FEMS Microbiol Lett 52:33-40

Williams RAD, da Costa MS (1992) The genus Thermus and related microorganisms. In: Balows A, Trüper HG, Dworkin M, Harder
W, Schleifer KH (eds) The prokaryotes, 2nd edn. Springer, Heidelberg, pp 3745-3753

Wolf A, Kramer R, Morbach S (2003) Three pathways for trehalose metabolism in Corynebacterium glutamicum ATCC13032 and their significance in response to osmotic stress. Mol Microbiol 49:1119-1134

Woodruff PJ, Carlson BL, Siridechadilok B, Pratt MR, Senaratne RH, Mougous JD, Riley LW, Williams SJ, Bertozzi CR (2004) Trehalose is required for growth of Mycobacterium smegmatis. J Biol Chem 279:28835-28843 